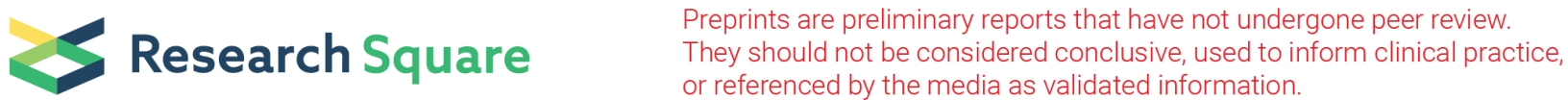

\section{Cardiovascular Disease in Women with Breast Cancer - A Nationwide Registry Study}

Marie Jakobsen ( $\nabla$ maja@vive.dk)

Nationale Forsknings- og Analysecenter for Velfard https://orcid.org/0000-0003-3068-1428

Christophe Kolodziejczyk

Nationale Forsknings- og Analysecenter for Velfard

Morten Sall Jensen

Nationale Forsknings- og Analysecenter for Velfard

Peter Bo Poulsen

Pfizer Denmark

Humma Khan

Pfizer Denmark

Thomas Kümler

Herlev Hospital

Michael Andersson

Rigshospitalet

Research article

Keywords: Breast cancer, cardiovascular disease, prevalence, incidence, cohort study, matched control group

Posted Date: December 2nd, 2020

DOI: https://doi.org/10.21203/rs.3.rs-113913/v1

License: (c) (i) This work is licensed under a Creative Commons Attribution 4.0 International License. Read Full License 


\section{Abstract}

Background: There is increasing concern about cardiovascular disease (CVD) after breast cancer (BC). The aim of this study was to estimate the prevalence of different types of CVD in women diagnosed with BC compared to cancer-free controls as well as the incidence of CVD after BC diagnosis.

Methods: We performed a cohort study based on data from national registries covering the entire Danish population. We followed 16,505 cancer-naïve BC patients diagnosed from 2003 to 2007 five years before and up to 10 years after BC diagnosis compared to 165,042 cancer-free controls.

Results: We found that $15.6 \%$ of $\mathrm{BC}$ patients were registered with at least one CVD diagnosis in hospital records before BC diagnosis. Overall, BC patients and controls were similar with regards to CVD comorbidity before BC diagnosis. After BC diagnosis, the incidence of all CVD diagnoses combined was significantly higher in $\mathrm{BC}$ patients than controls up to approximately 6 years after the index date (BC diagnosis). The incidence of heart failure, thrombophlebitis/thrombosis and pulmonary heart disease including pulmonary embolism remained higher in BC patients than controls during the entire 10 year follow-up period. Furthermore, we found that the risk of heart failure and thrombophlebitis/thrombosis was higher after chemotherapy.

Conclusions: Focus on CVD in BC patients is important to ensure optimum treatment with regards to BC as well as possible CVD. Strategies to minimise and manage the increased risk of heart failure, thrombophlebitis/thrombosis and pulmonary heart disease including pulmonary embolism in BC patients are especially important.

\section{Background}

Breast cancer (BC) is the most prevalent type of cancer among women worldwide [1]. In Denmark, approximately 4,700 women are diagnosed with BC every year, corresponding to $25 \%$ of all new primary cancer cases among women, and $10 \%$ of all women are diagnosed with $\mathrm{BC}$ before they become 75 years old [2].

Many patients have coexisting diseases (comorbidity) when they are diagnosed with cancer, especially elderly cancer patients [3]. International studies show that $20-35 \%$ of $\mathrm{BC}$ patients have one or more comorbidities at the time of BC diagnosis [4]. In Danish women diagnosed with early stage BC, the percentage with comorbidity has been shown to be approximately $20 \%[5,6]$.

Most existing studies assess comorbidity in BC patients using an aggregated measure such as the Charlson Comorbidity Index (CCI) [4-9]. Fewer studies provide information on the prevalence and incidence of specific comorbid conditions and different types of CVD in particular [10-12]. Such information is critical to inform clinicians and ensure optimum treatment of $\mathrm{BC}$ patients with regards to $\mathrm{BC}$ as well as possible CVD.

Abdel-Qadir et al. [11] find that women diagnosed with early stage BC are more likely to have a history of heart failure, ischemic heart disease, cerebrovascular disease, peripheral vascular disease, atrial fibrillation and hypertension compared to cancer-free controls, and that the incidence of heart failure, cerebrovascular disease and arrhythmias is significantly higher in BC patients up to 10 years after BC diagnosis. Strongman et al. [12] find that the incidence of heart failure, venous thromboembolism and pericarditis is significantly higher in BC survivors compared to cancer-free controls up to two years after BC diagnosis, but do not find a higher incidence of stroke or arrhythmias as in the study by Abdel-Qadir et al. Both studies are based on registry data covering a segment of the population in Canada and the UK, respectively. Even though the segment of the UK population (6.9\%) in the study by Strongman et al. has been shown to be broadly representative of the general UK population in terms of age, gender and ethnicity [13], a risk of selection bias remains as the study population can differ from the general population in other ways.

The overall aim of our study was to estimate the prevalence and incidence of different types of CVD in BC patients compared to cancer-free controls up to 5 years before and 10 years after $\mathrm{BC}$ diagnosis based on registry data covering the entire Danish population. Furthermore, we investigate the risk of developing CVD in BC patients following chemotherapy, radiation therapy, antibody therapy and hormonal therapy.

\section{Methods}

We performed a cohort study based on data from national registries covering the entire Danish population (5.8 million inhabitants). Data from the Danish Cancer Registry (CAR) [14], the Danish National Patient Registry (NPR) [15, 16], the Danish National Prescription Registry for Drugs (NPRD) [17, 18], Danish education registers [19] and the Danish Civil Registration System [20] were linked using the unique personal identification number assigned to every citizen in Denmark.

\section{Study population}

We used CAR to identify all women who were 18 + years old and diagnosed with BC in Denmark during the period from 1 January 2003 to 31 December 2007. CAR is a research registry containing information at the individual level on the incidence of cancer in the Danish population since 1943 [14]. Diagnoses in CAR are coded according to the International Classification of Diseases 7th Revision (ICD-7) from 1943 to 1978 and according to the International Classification of Diseases 10th Revision (ICD-10) from 1978 and onwards [14]. We used the diagnosis code C50* (ICD-10) to identify BC patients in the study population. Women who were registered in CAR with any cancer diagnosis (ICD-10: C00*-C97* or ICD-7: 140*-207*) prior to the BC diagnosis or who had lived outside Denmark for more than one year when they were $18+$ years old were excluded to allow focus on newly diagnosed BC patients who had no previous cancer diagnosis (cancer-naïve BC patients). The index date was that of BC diagnosis in CAR.

Subgroups of BC patients were defined according to age group ( $<45,45-74$ and $75+)$ and cancer disease stage (stage I-III, stage IV or unknown) at index date. Data on cancer disease stage at index date were obtained from CAR. 
Exact matching was used to identify a control group of women from the general population in Denmark without any cancer diagnosis in CAR (ICD-10: C00*C97* or ICD-7: $\left.140^{\star}-207^{\star}\right)$ up to and including the index year (i.e. the year of BC diagnosis of the matched BC patient). For each BC patient, we identified 10 controls matched according to age group (<30, 30-34, 35-39, 40-44, 45-49, 50-54, 55-59, 60-64, 65-69, 70-74, 75-80, 80-85, 85+), region of residence (Capital Region, Region Zealand, Region of Southern Denmark, Central Jutland Region, North Jutland Region) and education (low, medium, high, unknown). Each person in the control group was assigned a random index date during the index year.

\section{Outcomes and covariates}

We defined CVD as at least one primary or secondary CVD diagnosis in NPR, see Fig. 1. NPR holds information on all patients discharged from Danish hospitals since 1977 and on emergency department and outpatient visits since 1995 [16]. For each hospital contact, one primary and optional secondary diagnoses are registered according to the International Classification of Diseases (ICD). The primary diagnosis is the main reason for the hospital contact and secondary diagnoses identify other relevant diseases.

Furthermore, we identified BC patients with at least two CVD drug prescriptions (i.e. drugs related to CVD disease management) within the same year in NPRD, see Fig. 2. NPRD includes information on all primary care prescription drugs dispensed from community pharmacies in Denmark coded according to the Anatomical Therapeutic Chemical Classification System (ATC) [18]. We applied the criteria of two prescriptions to exclude persons who were apparently not in continuous treatment. It is not straightforward to identify CVD patients based on NPRD data as the drugs listed in Box 2 are not used exclusively for CVD disease management, and the NPRD does not contain the same information on diagnoses as NPR. However, NPRD data are still relevant to capture the large number of CVD patients who are not registered in NPR because their condition does not require hospitalization or outpatient visits to hospitals.

We used $\mathrm{CCl}$ as an aggregate measure of overall comorbidity [21] and diabetes at index date as covariates in multivariate Cox regression analyses. We calculated $\mathrm{CCl}$ using information from NPR on primary and secondary diagnoses up to five years before the index date. We classified the study population into three groups with a $\mathrm{CCl}$ score equal to zero, 1, or 2 and above, respectively. We defined diabetes at index date as at least one primary or secondary diabetes diagnosis in NPR (ICD-10: E10*-E11*) up to five years prior to the index date or at least two insulin prescriptions or prescriptions for blood glucose lowering drugs in NPRD (ATC group $A 10 A^{\star}$ and $A 10 B^{\star}$ ) within the same year during the 5-year period.

We identified persons in the BC group who had received different types of cancer treatments based on data from NPR. Surgery was defined by a 'surgery procedure code' in combination with BC diagnosis. Chemotherapy was defined by a BWHA procedure code, radiation therapy was defined by a BWG procedure code, antibody therapy was defined by a BOHJI procedure code, and hormonal therapy was defined by a BWHC procedure code

We identified persons in the $\mathrm{BC}$ and control group who died during follow-up (including date of death) based on data from the Civil Registration System.

Data on age, region of residence and education were obtained from the Civil Registration System and education registers.

\section{Statistical analysis}

CVD prevalence rates were calculated as number of persons with at least one CVD primary or secondary diagnosis in NPR up to five years prior to the index date per 1,000 persons for all CVD diagnoses combined and specific types of CVD. Furthermore, number of BC patients and cancer-free controls treated with CVD prescription drugs up to five years prior to the index date per 1,000 persons were calculated based on NPRD data.

The cumulative incidence of all CVD diagnoses combined as well as specific types of CVD were calculated up to 10 years after the index date (BC diagnosis) allowing for the presence of competing risk of death [22]. A competing risk is an event whose occurrence precludes another event of interest.

We performed multivariate Cox regression analyses to investigate the risk of developing CVD in BC patients following chemotherapy, radiation therapy, antibody therapy and hormonal therapy initiated within nine months after BC diagnosis. The risk of CVD was calculated based on either CVD diagnoses in NPR (model 1) or CVD prescription drugs in NPDR (model 2). Only BC patients without any CVD diagnosis in NPR five years prior to the index date were included in model 1 , and only BC patients without a history of CVD prescription drugs five years prior to the index date were included in model 2 . We included time dependent exposure dummies that classified the patients' time-to-event' as unexposed until treatment began to avoid immortal time bias [23]. BC patients who had received surgery, but not the cancer treatment in question (chemotherapy, radiation therapy, antibody therapy or hormonal therapy) were used as reference. Adjustments were made for differences between groups with regards to age, region of residence, education and cohort (year of BC diagnosis) as well as comorbidity. Persons who died during follow-up were censored at time of death.

The analyses were performed using Stata 14 (StataCorp, College Station, TX). The statistical significance of differences between groups were evaluated using two-sided t-tests with a $5 \%$ level of significance or $95 \%$ confidence intervals.

\section{Results}

The study population included 16,505 BC patients and 165,042 cancer-free controls, see Table 1 . By matching design, BC patients and controls were identical with regards to age, region of residence, and education as well as gender (they were all women). 
Table 1

Baseline characteristics of BC patients and cancer-free controls

BC patients

\begin{tabular}{lllll}
$\begin{array}{l}\text { Stage } \leq \text { III (diagnosed without distant } \\
\text { metastasis) }\end{array}$ & $\begin{array}{l}\text { Stage }=\text { IV (diagnosed with distant } \\
\text { metastasis) }\end{array}$ & $\begin{array}{l}\text { Stage } \\
\text { unknown }\end{array}$ & Total & controls \\
\hline $\mathrm{N}=12,715$ & $\mathrm{~N}=997$ & $\mathrm{~N}=2,793$ & $\begin{array}{l}\mathrm{N}= \\
16,505\end{array}$ & $\mathrm{~N}=165,042$ \\
\end{tabular}

\begin{tabular}{|c|c|c|c|c|c|}
\hline \multicolumn{6}{|l|}{ Age group } \\
\hline$<45$ & $11 \%$ & $6 \%$ & $7 \%$ & $10 \%$ & $10 \%$ \\
\hline $45-54$ & $21 \%$ & $12 \%$ & $16 \%$ & $20 \%$ & $20 \%$ \\
\hline $55-64$ & $31 \%$ & $25 \%$ & $23 \%$ & $29 \%$ & $29 \%$ \\
\hline $65-74$ & $21 \%$ & $27 \%$ & $19 \%$ & $21 \%$ & $21 \%$ \\
\hline $75+$ & $15 \%$ & $29 \%$ & $35 \%$ & $19 \%$ & $19 \%$ \\
\hline \multicolumn{6}{|c|}{ Region of residence } \\
\hline $\begin{array}{l}\text { Northern } \\
\text { Jutland }\end{array}$ & $10 \%$ & $10 \%$ & $10 \%$ & $10 \%$ & $10 \%$ \\
\hline Central Jutland & $21 \%$ & $22 \%$ & $19 \%$ & $20 \%$ & $20 \%$ \\
\hline $\begin{array}{l}\text { Southern } \\
\text { Denmark }\end{array}$ & $23 \%$ & $19 \%$ & $26 \%$ & $23 \%$ & $23 \%$ \\
\hline Capital & $30 \%$ & $29 \%$ & $31 \%$ & $30 \%$ & $30 \%$ \\
\hline Zealand & $16 \%$ & $20 \%$ & $15 \%$ & $16 \%$ & $16 \%$ \\
\hline \multicolumn{6}{|l|}{ Education } \\
\hline Low & $38 \%$ & $49 \%$ & $37 \%$ & $38 \%$ & $38 \%$ \\
\hline Medium & $39 \%$ & $30 \%$ & $32 \%$ & $37 \%$ & $37 \%$ \\
\hline High & $19 \%$ & $11 \%$ & $14 \%$ & $18 \%$ & $18 \%$ \\
\hline Unknown & $4 \%$ & $10 \%$ & $17 \%$ & $6 \%$ & $6 \%$ \\
\hline \multicolumn{6}{|c|}{ Cancer treatment started within 9 months from BC diagnosis } \\
\hline Surgery & $96 \%$ & $63 \%$ & $81 \%$ & $91 \%$ & N.a. \\
\hline Chemotherapy & $35 \%$ & $37 \%$ & $22 \%$ & $33 \%$ & N.a. \\
\hline $\begin{array}{l}\text { Radiation } \\
\text { therapy }\end{array}$ & $62 \%$ & $28 \%$ & $34 \%$ & $55 \%$ & N.a. \\
\hline $\begin{array}{l}\text { Antibody } \\
\text { therapy }\end{array}$ & $4 \%$ & $7 \%$ & $2 \%$ & $4 \%$ & N.a. \\
\hline $\begin{array}{l}\text { Hormonal } \\
\text { therapy }\end{array}$ & $41 \%$ & $38 \%$ & $26 \%$ & $39 \%$ & N.a. \\
\hline
\end{tabular}

Of the 16,505 BC patients, 12,715 (77\%) were diagnosed with early stage BC (i.e. stage I-III without distant metastasis) and 997 (6\%) were diagnosed in stage IV (i.e. with distant metastasis), see Table 1. For the remaining 2,793 BC patients (17\%), cancer disease stage at the time of diagnosis was unknown. Almost all BC patients received surgery (91\%), 55\% received radiation therapy, 39\% received hormonal therapy, $33 \%$ received chemotherapy, and $4 \%$ received antibody therapy initiated within nine months of BC diagnosis. A larger proportion of BC patients diagnosed in stage I-III received surgery, radiation therapy and hormonal therapy compared to BC patients diagnosed in stage IV.

In total, 156 per 1,000 BC patients (15.6\%) were registered with at least one CVD diagnosis in hospital records up to five years before the index date (BC diagnosis) compared to 151 per 1,000 cancer-free controls (15.1\%), see Table 2 . The most common type of CVD diagnosis was hypertension (7.3\% of BC patients compared to $7.0 \%$ of controls) followed by ischemic heart disease/acute coronary syndrome (4.5\% of BC patient compared to $4.7 \%$ of controls) and cerebrovascular disease (3.7\% of BC patients compared to $3.6 \%$ of controls). The prevalence rates of CVD were not significantly different between BC patients and controls with the exception of atrial fibrillation $(p=0.0019)$, which was higher in BC patients $(3.2 \%$ compared to $2.6 \%$ in controls). 
Table 2

Number of persons with CVD diagnosis before the index date per 1,000 persons

\begin{tabular}{|lllll|}
\hline & BC patients & Cancer-free controls & Difference & P value \\
\hline CVD & 156.3 & 150.7 & 5.6 & 0.0546 \\
\hline Hypertension & 73.4 & 69.6 & 3.8 & 0.0687 \\
\hline Ischemic heart disease/ACS & 44.9 & 47.3 & -2.4 & 0.1659 \\
\hline Cerebrovascular disease & 36.5 & 36.4 & 0.1 & 0.9409 \\
\hline Atrial fibrillation & 30.2 & 26.1 & $4.1 * *$ & 0.0019 \\
\hline Heart failure & 21.4 & 20.1 & 1.4 & 0.2251 \\
\hline Heart valve disease & 9.0 & 9.1 & 0.0 & 0.9745 \\
\hline Thrombophlebitis/thrombosis & 8.5 & 7.6 & 0.9 & 0.2017 \\
\hline Other cardiac arrhythmias & 8.2 & 7.3 & 0.9 & 0.2189 \\
\hline Peripheral vascular disease & 6.7 & 7.7 & -1.1 & 0.1272 \\
\hline Pulmonary heart disease & 4.7 & 4.0 & 0.7 & 0.1907 \\
\hline${ }^{*} p<0.05,{ }^{* *} p<0.01{ }^{* * *} p<0.001$ & & & \\
\hline
\end{tabular}

In total, 447 per 1,000 BC patients (44.7\%) had a history of CVD drug prescriptions (i.e. drugs related to CVD disease management) when diagnosed with BC compared to 445 per 1,000 cancer-free controls (44.5\%). Diuretics were the most common type of drug used by both BC patients and controls ( $20 \%$ ) followed by agents acting on the renin-angiotensin system (14\%) and beta blocking agents (11\%). There was no significant differences between BC patients and controls (data not shown).

Up to approximately 6 years after the index date, the cumulative incidence of all CVD diagnoses combined was higher in BC patients than in cancer-free controls taking account of the presence of competing risk of death, see Fig. 3. Hereafter, the difference in cumulative CVD incidence between BC patients and controls was no longer statistically significant. After 10 years, $28 \%$ of both BC patients and controls (without any CVD diagnosis in NPR up to five years before the index date) had at least one CVD diagnosis in NPR. The most common types of CVD diagnosis were hypertension with a cumulative incidence of $15 \%$ after 10 years followed by ischemic heart disease/acute coronary syndrome, cerebrovascular disease and atrial fibrillation (all with a cumulative incidence of $5 \%$ after 10 years).

During the first 2 years after the index date, the cumulative incidence of most types of CVD was statistically significantly higher in BC patients than controls, see Fig. 4. However, only the cumulative incidence of heart failure, thrombophlebitis/thrombosis and pulmonary heart disease including pulmonary embolism remained higher in BC patients during the entire 10-year follow-up period. After 10 years, $2.7 \%$ of BC patients compared to $2.5 \%$ of controls had developed heart failure, $2.7 \%$ of $\mathrm{BC}$ patients compared to $1.5 \%$ of controls had developed thrombophlebitis/thrombosis, and $1.5 \%$ of $\mathrm{BC}$ patients compared to $1.0 \%$ of controls had developed pulmonary heart disease.

The risk of CVD was significantly elevated in BC patients after chemotherapy (HR >1), no matter whether CVD was defined according to CVD diagnoses registered in NPR (95\% Cl: 1.030-1.353), see Table 3 (model 1), or CVD drug prescriptions registered in NPRD (95\% Cl: 1.226-1.520), see Table 3 (model 2). Furthermore, the risk of CVD was significantly higher after radiation therapy (HR $>1)$, when CVD was defined according to CVD drug prescriptions in NPDR (95\% Cl: 1.023-1.251), but not CVD diagnoses in NPR (95\% Cl: $0.887-1.117)$. There were no statistically significant results regarding the risk of CVD after hormonal or antibody therapy. 
Table 3

Hazard ratio (HR) of CVD in BC patients after different types of cancer treatment

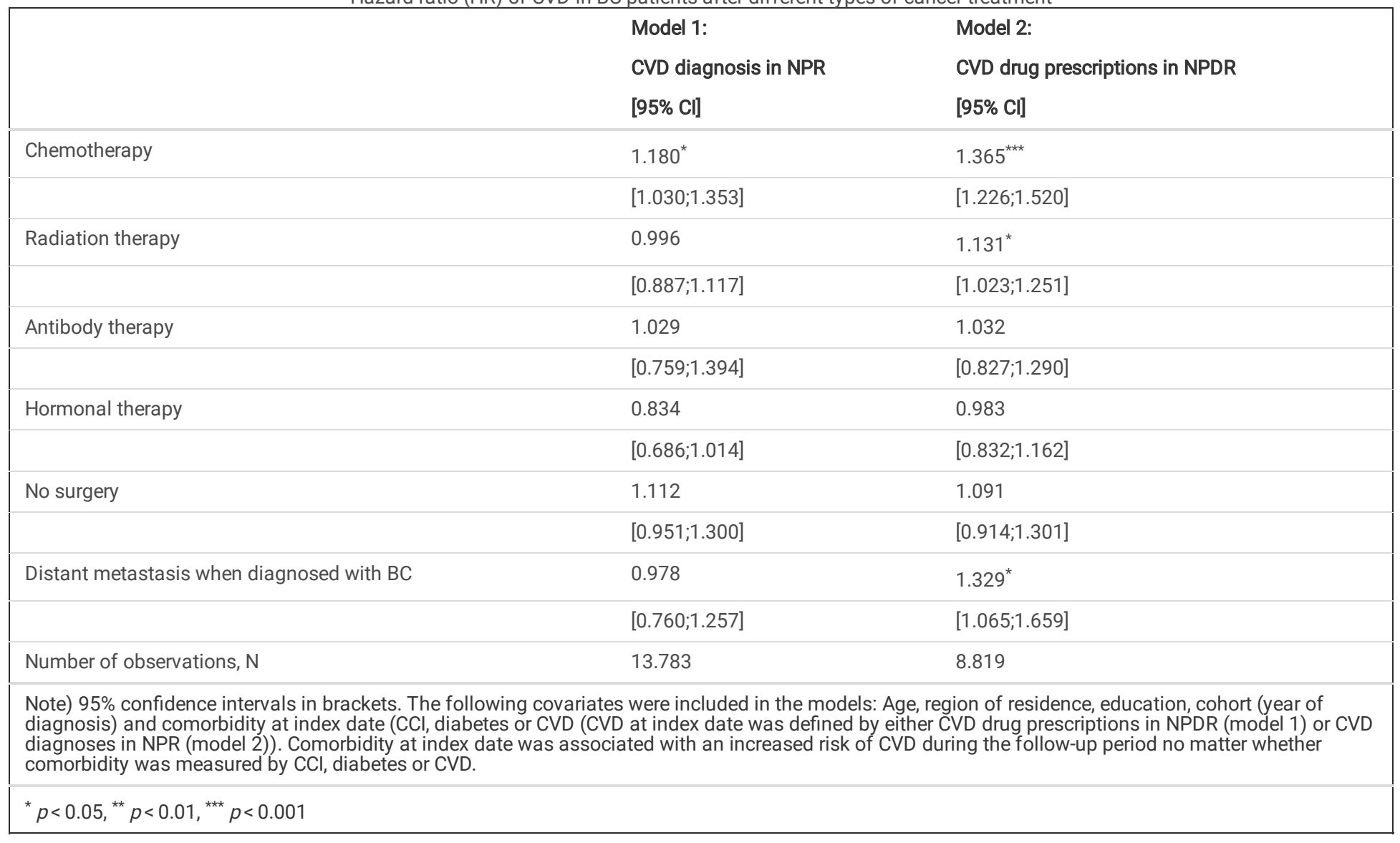

When investigating the risk of different types of CVD after chemotherapy, we found that the risk of heart failure and thrombophlebitis/thrombosis was significantly elevated in BC patients who received chemotherapy compared to BC patients who did not, see Table 4. Furthermore, we found that the differences in risk of developing heart failure and thrombophlebitis/thrombosis increased during the 10-year follow-up period, see Fig. 5.

Table 4

Hazard ratio (HR) of different types of CVD in BC patients after chemotherapy

\begin{tabular}{|lllllllll}
\hline & Hypertension & $\begin{array}{l}\text { Cerebrovascular } \\
\text { disease }\end{array}$ & $\begin{array}{l}\text { Ischemic } \\
\text { heart } \\
\text { disease/Acs }\end{array}$ & $\begin{array}{l}\text { Atrial } \\
\text { fibrillation }\end{array}$ & $\begin{array}{l}\text { Heart } \\
\text { failure }\end{array}$ & Thrombophlebitis/thrombosis & $\begin{array}{l}\text { Pulmonary } \\
\text { heart } \\
\text { disease }\end{array}$ & $\begin{array}{l}\text { Peripheral } \\
\text { vascular } \\
\text { disease }\end{array}$ \\
\hline Chemotherapy & 0.849 & 1.012 & 1.004 & 1.189 & $1.738^{* *}$ & $1.737^{* *}$ & 1.408 \\
$\begin{array}{l}\text { Number of } \\
\text { observations, }\end{array}$ & 15,574 & 16,000 & 15,874 & 16,080 & 16,245 & 16,387 & 1692 \\
\hline
\end{tabular}

$\mathrm{N}$

Note) The following covariates were included in the model: Age, region of residence, education, cohort (year of diagnosis), distant metastasis when diagnose comorbidity at index date (CCl, diabetes or CVD (CVD at index date was defined by CVD drug prescriptions in NPDR

${ }^{\star} p<0.05,{ }^{* \star} p<0.01,{ }^{* \star *} p<0.001$

\section{Discussion}

We found that $15.6 \%$ of $\mathrm{BC}$ patients were registered with at least one CVD diagnosis in hospital records up to five years before BC diagnosis, and a considerably higher percentage of patients had a history of CVD drug prescriptions (i.e. drugs related to CVD disease management). Overall, BC patients and cancer-free controls were similar with regards to CVD comorbidity at index date. The most common types of CVD diagnoses in both BC patients and controls were hypertension, ischemic heart disease/acute coronary syndrome, and cerebrovascular disease.

After $\mathrm{BC}$ diagnosis, the incidence of CVD was significantly higher in BC patients than controls for all CVD diagnoses combined up to approximately 6 years after the index date (BC diagnosis) taking account of the presence of competing risk of death. After 10 years, $28 \%$ of both $\mathrm{BC}$ patients and controls (without any CVD diagnosis in NPR five years before the index date) had at least one CVD diagnosis in NPR. Only the cumulative incidence of heart failure, thrombophlebitis/thrombosis and pulmonary heart disease including pulmonary embolism remained higher in BC patients than controls during the entire 10year follow-up period. Furthermore, the risk of heart failure and thrombophlebitis/thrombosis was significantly higher in BC patients who had received chemotherapy compared to BC patients who had not as well as cancer-free controls. Heart failure has been associated with chemotherapy in previous studies 
[24], and it is well established that cancer patients have increased risk of thrombotic complications including e.g. deep vein thrombosis, pulmonary embolism and arterial thrombosis [25].

Contrary to our results, Abel-Qadir et al. [11] found that the prevalence of different types of CVD was significantly higher in BC patients compared to cancerfree controls. A possible explanation for this discrepancy could be that Abel-Qadir et al. used different algorithms to determine the presence of CVD preceding the index date. Other possible explanations are differences in the definition of the study population and matching criteria, as Abel-Qadir et al. included women diagnosed with early stage BC only, and controls were matched to BC patients according to gender and age, but not education as in our study.

Abel-Qadir et al. [11] found a significantly higher incidence of CVD hospitalizations due to heart failure, cerebrovascular disease and arrhythmias in BC patients compared to cancer-free controls whereas we did not find this for cerebrovascular disease or arrhythmias. In accordance with our results, Strongman et al. [12] found a statistically significantly higher incidence of heart failure and venous thromboembolism in BC patients compared to cancer-free controls, but not significant differences in the incidence of arrhythmia, stroke or peripheral vascular disease. Furthermore, Strongman et al. found that the incidence of coronary artery disease was significantly lower in BC patients compared to controls.

Existing studies have shown an elevated risk of CVD in BC patients after anthracycline-based chemotherapy, radiation therapy and antibody therapy [11, 2628]. Our study confirms an elevated risk of CVD after chemotherapy. Unfortunately, data available for our study did not allow distinction between different types of chemotherapy. We also saw a tendency towards a higher risk of CVD after radiation and antibody therapy, but these results were in general not statistically significant.

Hormonal agents like tamoxifen approved for BC treatment more than 30 years ago can have both beneficial and detrimental effects on the cardiovascular system [24]. Studies have shown that tamoxifen has a protective effect on lipid metabolism [29-31], but at the same time increases the risk of venous thrombosis and thromboembolism [32]. In our study, we did not find a higher risk of CVD after hormonal therapy, but we see a higher incidence of thrombotic complications in $\mathrm{BC}$ patients compared to cancer-free controls.

The present study has several strengths. Firstly, it is a study at the population level based on a large real-world dataset with no selection bias or other potential issues related to more segmented and selective populations. Secondly, the study population was identified from CAR, which has high completeness and validity due to use of notifications from different data sources and quality control [14]. Thirdly, we used prescription data from NPRD to identify CVD patients as a supplement to data from NPR, as the latter registry does not include patients who require primary care only. NPRD is the national registry of prescription drugs dispensed from community pharmacies and is considered both complete and valid from 1995 and onwards [18]. Fourthly, the study included a cancerfree control group, and the use of exact matching ensured that $\mathrm{BC}$ patients and controls were comparable with regards to age, region of residence, and education as well as gender (they were all women). We did not include $\mathrm{CCl}$ as a matching criteria to avoid overmatching, and we preferred exact matching to propensity score matching because exact matching ensures that groups are identical with regard to the matching criteria used. Finally, we allowed for the presence of competing risk of death when estimating the cumulative incidence function [22, 33]. Since BC patients had a higher risk of dying during the 10year follow-up period than controls, ignoring the presence of competing risk could result in substantial bias.

The study also has limitations. The study relies on diagnosis and procedure codes in NPR, which may contain errors or be incomplete, e.g. many doctors code the primary diagnosis only, even if the patient has other relevant diseases [34]. Furthermore, NPR does contain information on patients who require primary care only as mentioned above, and it is not straightforward to identify CVD patients based on NPRD data as prescription drugs used for CVD disease management may have other indications. Finally, there is a risk of confounding due to the observational nature of the study. The risk of confounding related to observable baseline characteristics was minimised by exact matching and a multivariable regression design, but BC patients and controls may differ on nonobservable lifestyle factors, that influence the risk of CVD. However, we expect lifestyle factors to be similar in BC patients and controls because education is a proxy for lifestyle and was included as a matching criteria. Similar comorbidity at index date also indicate a comparable lifestyle between BC patients and controls.

\section{Conclusions}

In conclusion, we found that $15.6 \%$ of BC patients were registered with at least one CVD diagnosis in hospital records when diagnosed with BC. Overall, BC patients and controls were similar with regards to CVD comorbidity before BC diagnosis. After BC diagnosis, the incidence of all CVD diagnoses combined was significantly higher in $\mathrm{BC}$ patients than controls up to approximately 6 years after the index date. The incidence of heart failure, thrombophlebitis/thrombosis and pulmonary heart disease including pulmonary embolism remained higher in BC patients than controls during the entire 10 year follow-up period. Strategies to minimise and manage the increased risk of CVD in BC patients are important.

\section{Abbreviations}

ATC: Anatomical Therapeutic Chemical Classification System; BC: Breast cancer; CAR: The Danish Cancer Registry; CCI: Charlson Comorbidity Index; 95\% Cl: 95\% confidence interval; CVD: Cardiovascular disease; HR: Hazard ratio; ICD: International Classification of Diseases; NPRD: Danish Prescription Drug Registry; NPR: the Danish National Patient Registry.

\section{Declarations}

Ethics approval and consent to participate 
Neither informed consent from patients nor approval from an ethics committee was required by Danish law since the study was based on retrospective registry data only. Research Ethics Committees in Denmark assess only biomedical research.

The study was carried out in accordance with international ethical standards including the EU general data protection regulation. Data for statistical analyses were anonymized, and study results were reported so that it is not possible to identify individual persons.

Consent for publication

Not applicable.

Availability of data and materials

The data that support the findings of this study are available from the Danish Health Data Authority and Statistics Denmark but restrictions apply to the availability of these data, which were used under license for the current study, and so are not publicly available.

Competing interests

MJ, CK and MSJ report grants from Pfizer Denmark during the conduct of the study. TK and MA report personal fees from Pfizer Denmark during the conduct of the study. PBP and HK report personal fees from Pfizer Denmark during the conduct of the study and own shares in Pfizer Inc. outside submitted work.

MJ, CK and MSJ are employees of the National Center for Social Science Research (VIVE), which was a paid vendor to Pfizer Denmark on the project. VIVE is an independent research institute, which is under obligation by law to disseminate the results of its work to relevant public and private stakeholders and the public in general. MA and TK are medical doctors with expertise in breast cancer and cardiovascular disease, respectively. PBP and HK are employees of Pfizer Denmark.

Funding

The study was funded by Pfizer Denmark.

Authors' contribution

MJ, CK, MSJ, PBP, HK, TK and MA were all involved in the conception and design of the study. CK and MSJ had the main responsibility for carrying out the statistical analyses, and MJ had the main responsibility for drafting the article. All authors were involved in the analysis and interpretation of data, revised the article critically and approved the final version for publication.

Acknowledgements

Not applicable.

\section{References}

1. [https://gco.iarc.fr/today/data/factsheets/populations/900-world-fact-sheets.pdf].

2. http://www-dep.iarc.fr/NORDCAN/DK/frame.asp.

3. Jorgensen TL, Hallas J, Friis S, Herrstedt J. Comorbidity in elderly cancer patients in relation to overall and cancer-specific mortality. Br J Cancer. 2012;106(7):1353-60.

4. Søgaard M, Thomsen RW, Bossen KS, Sørensen HT, Nørgaard M. The impact of comorbidity on cancer survival: a review. Clin Epidemiol. 2013;5(Suppl 1):3-29.

5. Statens Serum Institut. Sammenhæng mellem komorbiditet og behandling inden for standardforløbstiderne i pakkeforløb for kræft. København: Statens Serum Institut \& National Sundhedsdokumentation og -IT; 2015.

6. Land LH, Dalton SO, Jensen MB, Ewertz M. Impact of comorbidity on mortality: a cohort study of 62,591 Danish women diagnosed with early breast cancer, 1990-2008. Breast Cancer Research and Treatment 2012, 131(3):1013-1020.

7. Berglund A, Wigertz A, Adolfsson J, Ahlgren J, Fornander T, Warnberg F, Lambe M. Impact of comorbidity on management and mortality in women diagnosed with breast cancer. Breast Cancer Res Treat. 2012;135(1):281-9.

8. O'Connor T, Edge S, Kossoff E, Groman A, Wilding G, Ademiyiwa F, Levine EG, Watroba N, Ngamphaiboon N. Factors affecting the delivery of adjuvant/neoadjuvant chemotherapy in older women with breast cancer. Journal of Geriatric Oncology. 2012;3(4):320-8.

9. Punglia RS, Saito AM, Neville BA, Earle CC, Weeks JC. Impact of interval from breast conserving surgery to radiotherapy on local recurrence in older women with breast cancer: retrospective cohort analysis. BMJ. 2010;340:c845.

10. Ewertz M, Land LH, Dalton SO, Cronin-Fenton D, Jensen MB. Influence of specific comorbidities on survival after early-stage breast cancer. Acta Oncol. 2018;57(1):129-34.

11. Abdel-Qadir H, Thavendiranathan P, Austin PC, Lee DS, Amir E, Tu JV, Fung K, Anderson GM. The Risk of Heart Failure and Other Cardiovascular Hospitalizations after Early Stage Breast Cancer: A Matched Cohort Study. J Natl Cancer Inst. 2019;111(8):854-62.

12. Strongman H, Gadd S, Matthews A, Mansfield KE, Stanway S, Lyon AR, Dos-Santos-Silva I, Smeeth L, Bhaskaran K. Medium and long-term risks of specific cardiovascular diseases in survivors of 20 adult cancers: a population-based cohort study using multiple linked UK electronic health records databases. 
Lancet. 2019;394(10203):1041-54.

13. Herrett E, Gallagher AM, Bhaskaran K, Forbes H, Mathur R, van Staa T, Smeeth L. Data Resource Profile: Clinical Practice Research Datalink (CPRD). Int J Epidemiol. 2015;44(3):827-36.

14. Gjerstorff ML. The Danish Cancer Registry. Scand J Public Health. 2011;39(Suppl 7):42-5.

15. Lynge E, Sandegaard JL, Rebolj M. The Danish National Patient Register. Scand J Public Health. 2011;39(7 Suppl):30-3.

16. Schmidt M, Schmidt SA, Sandegaard JL, Ehrenstein V, Pedersen L, Sorensen HT. The Danish National Patient Registry: a review of content, data quality, and research potential. Clin Epidemiol. 2015;7:449-90.

17. Kildemoes HW, Sorensen HT, Hallas J. The Danish National Prescription Registry. Scand J Public Health. 2011;39(7 Suppl):38-41.

18. Pottegard A, Schmidt SAJ, Wallach-Kildemoes H, Sorensen HT, Hallas J, Schmidt M. Data Resource Profile: The Danish National Prescription Registry. Int J Epidemiol. 2017;46(3):798-8 f.

19. Jensen VM, Rasmussen AW. Danish Education Registers. Scand J Public Health. 2011;39(7 Suppl):91-4.

20. Pedersen CB. The Danish Civil Registration System. Scand J Public Health. 2011;39(7 Suppl):22-5.

21. Charlson ME, Pompei P, Ales KL, MacKenzie CR. A new method of classifying prognostic comorbidity in longitudinal studies: development and validation. Journal of Chronic Diseases. 1987;40(5):373-83.

22. Austin PC, Lee DS, Fine JP. Introduction to the Analysis of Survival Data in the Presence of Competing Risks. Circulation: Cardiovascular Quality Outcomes. 2016;133(6):601-9.

23. Suissa S. Immortal time bias in observational studies of drug effects. Pharmacoepidemiol Drug Saf. 2007;16(3):241-9.

24. Mehta LS, Watson KE, Barac A, Beckie TM, Bittner V, Cruz-Flores S, Dent S, Kondapalli L, Ky B, Okwuosa T, Pina IL, Volgman AS, American Heart Association Cardiovascular Disease in Women and Special Populations Committee of the Council on Clinical Cardiology. Council on Cardiovascular and Stroke Nursing, and Council on Quality of Care and Outcomes Research: Cardiovascular Disease and Breast Cancer. Where These Entities Intersect: A Scientific Statement From the American Heart Association. Circulation. 2018;137(8):e30-66.

25. Elyamany G, Alzahrani AM, Bukhary E. Cancer-associated thrombosis: an overview. Clin Med Insights Oncol. 2014;8:129-37.

26. Darby SC, Ewertz M, McGale P, Bennet AM, Blom-Goldman U, Brønnum D, Correa C, Cutter D, Gagliardi G, Gigante B, Jensen MB, Nisbet A, Peto R, Rahimi K, Taylor C, Hall P. Risk of ischemic heart disease in women after radiotherapy for breast cancer. N Engl J Med. 2013;368(11):987-98.

27. McGale P, Darby SC, Hall P, Adolfsson J, Bengtsson NO, Bennet AM, Fornander T, Gigante B, Jensen MB, Peto R, Rahimi K, Taylor CW, Ewertz M. Incidence of heart disease in 35,000 women treated with radiotherapy for breast cancer in Denmark and Sweden. Radiother Oncol. 2011;100(2):167-75.

28. Rehammar JC, Jensen MB, McGale P, Lorenzen EL, Taylor C, Darby SC, Videbæk L, Wang Z, Ewertz M. Risk of heart disease in relation to radiotherapy and chemotherapy with anthracyclines among 19,464 breast cancer patients in Denmark, 1977-2005. Radiother Oncol. 2017;123(2):299-305.

29. Dewar JA, Horobin JM, Preece PE, Tavendale R, Tunstall-Pedoe H, Wood RA. Long term effects of tamoxifen on blood lipid values in breast cancer. BMJ 1992, 305(6847):225-226.

30. Grey AB, Stapleton JP, Evans MC, Reid IR. The effect of the anti-estrogen tamoxifen on cardiovascular risk factors in normal postmenopausal women. J Clin Endocrinol Metab. 1995;80(11):3191-5.

31. Esteva FJ, Hortobagyi GN. Comparative assessment of lipid effects of endocrine therapy for breast cancer: implications for cardiovascular disease prevention in postmenopausal women. Breast. 2006;15(3):301-12.

32. Saphner T, Tormey DC, Gray R. Venous and arterial thrombosis in patients who received adjuvant therapy for breast cancer. J Clin Oncol. 1991;9(2):28694.

33. Abdel-Qadir H, Fang J, Lee DS, Tu JV, Amir E, Austin PC, Anderson GM. Importance of Considering Competing Risks in Time-to-Event Analyses: Application to Stroke Risk in a Retrospective Cohort Study of Elderly Patients With Atrial Fibrillation. Circulation: Cardiovascular Quality Outcomes. 2018;11(7):1-11.

34. Kumler T, Gislason GH, Kirk V, Bay M, Nielsen OW, Kober L, Torp-Pedersen C. Accuracy of a heart failure diagnosis in administrative registers. Eur J Heart Fail. 2008;10(7):658-60.

\section{Figures}


- Heart valve disease (ICD-10: I05.0-I05.1, I34.0-I34.2, I34.9-I35.1, I35.9)

- Hypertension (ICD-10: I10-I15 excl. I11.0, I13.0, I13.2)

- Ischemic heart disease and acute coronary syndrome (ACS)

- Angina pectoris (ICD-10: I20)

- Acute myocardial infarction (ICD-10: I21, I22, I25.2)

- Chronic ischemic heart disease (ICD-10: I25 excl. I25.2)

- Pulmonary heart disease and diseases of pulmonary circulation, including pulmonary embolism, (ICD-10: I26-I28)

- Atrial fibrillation (ICD-10: I48)

- Other cardiac arrhythmias (ICD-10: I49)

- Heart failure including cardiomyopathy and hypotensive heart (and renal) disease with heart (and renal) failure (ICD-10: I50, I42.0, I42.6, I42.7, I42.9, I11.0, I13.0, I13.2)

- Cerebrovascular disease (I60-I69, G45-G46)

- Peripheral vascular disease (I71, I79.0, I73.9, R02, Z95.8, Z95.9))

- Thrombophlebitis and thrombosis (ICD10: I80-I82)

Figure 1

Types of CVD included in the study (ICD-10 diagnoses)

- Heart valve disease (ICD-10: I05.0-I05.1, I34.0-I34.2, I34.9-I35.1, I35.9)

- Hypertension (ICD-10: I10-I15 excl. I11.0, I13.0, I13.2)

- Ischemic heart disease and acute coronary syndrome (ACS)

- Angina pectoris (ICD-10: I20)

- Acute myocardial infarction (ICD-10: I21, I22, I25.2)

- Chronic ischemic heart disease (ICD-10: I25 excl. I25.2)

- Pulmonary heart disease and diseases of pulmonary circulation, including pulmonary embolism, (ICD-10: I26-I28)

- Atrial fibrillation (ICD-10: I48)

- Other cardiac arrhythmias (ICD-10: I49)

- Heart failure including cardiomyopathy and hypotensive heart (and renal) disease with heart (and renal) failure (ICD-10: I50, I42.0, I42.6, I42.7, I42.9, I11.0, I13.0, I13.2)

- Cerebrovascular disease (I60-I69, G45-G46)

- Peripheral vascular disease (I71, I79.0, I73.9, R02, Z95.8, Z95.9))

- Thrombophlebitis and thrombosis (ICD10: I80-I82)

Figure 1

Types of CVD included in the study (ICD-10 diagnoses)

- B01. ANTITHROMBOTIC AGENTS

- C01B. ANTI-ARYTMICA

- C02. ANTIHYPERTENSIVES

- C03. DIURETICS

- C04. PERIPHERAL VASODILATORS

- C05. VASOPROTECTIVES

- C07. BETA BLOCKING AGENTS

- C08. CALCIUM CHANNEL BLOCKERS

- C09. AGENTS ACTING ON THE RENIN-ANGIOTENSIN SYSTEM

- C10. LIPID MODIFYING AGENTS 
Figure 2

CVD prescription drugs included in the study (ATC groups)

- B01. ANTITHROMBOTIC AGENTS

- C01B. ANTI-ARYTMICA

- C02. ANTIHYPERTENSIVES

- C03. DIURETICS

- C04. PERIPHERAL VASODILATORS

- C05. VASOPROTECTIVES

- C07. BETA BLOCKING AGENTS

- C08. CALCIUM CHANNEL BLOCKERS

- C09. AGENTS ACTING ON THE RENIN-ANGIOTENSIN SYSTEM

- C10. LIPID MODIFYING AGENTS

Figure 2

CVD prescription drugs included in the study (ATC groups)

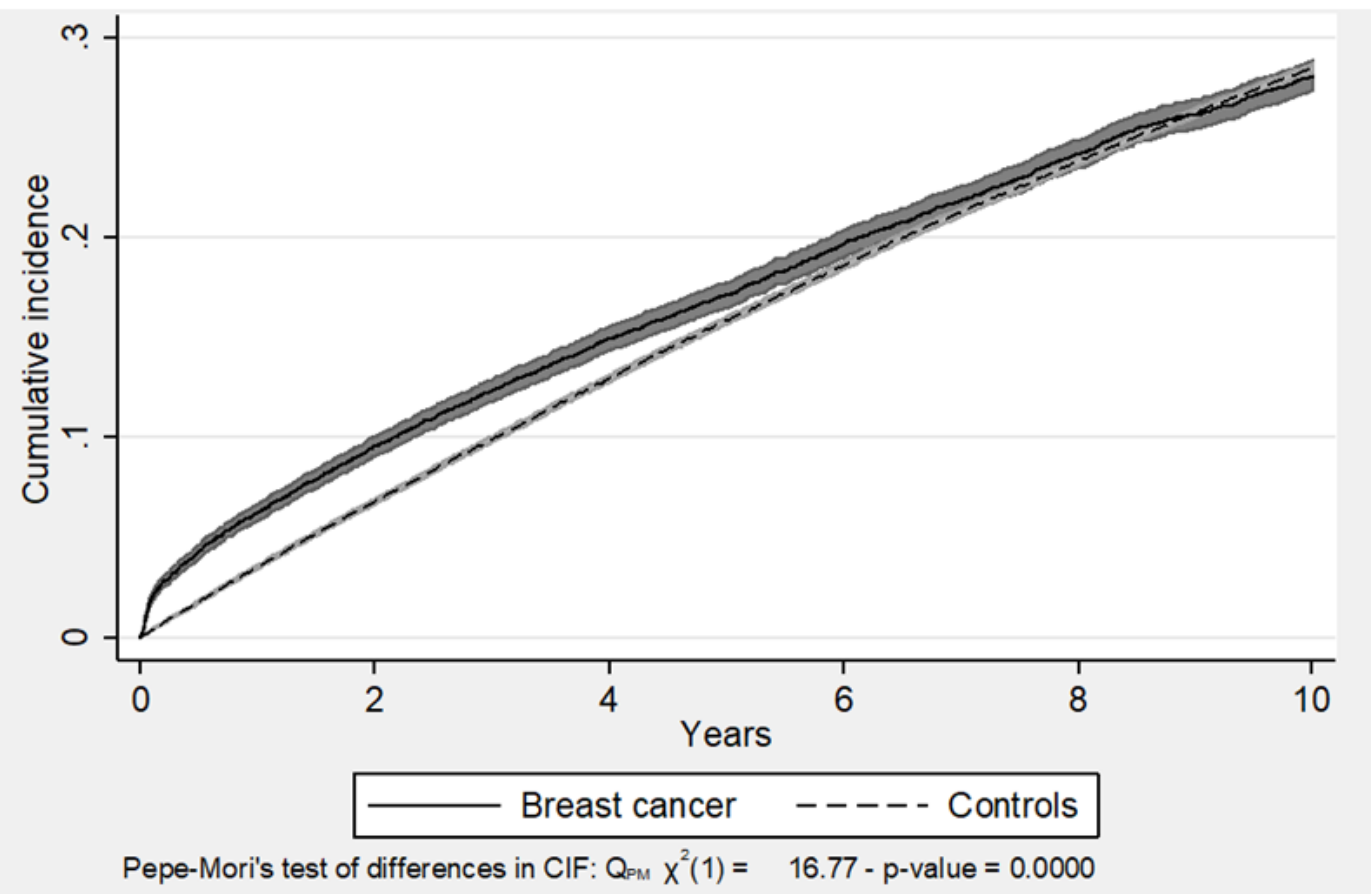

Figure 3

Cumulative incidence of all CVD diagnoses combined, proportion with at least one CVD diagnosis 


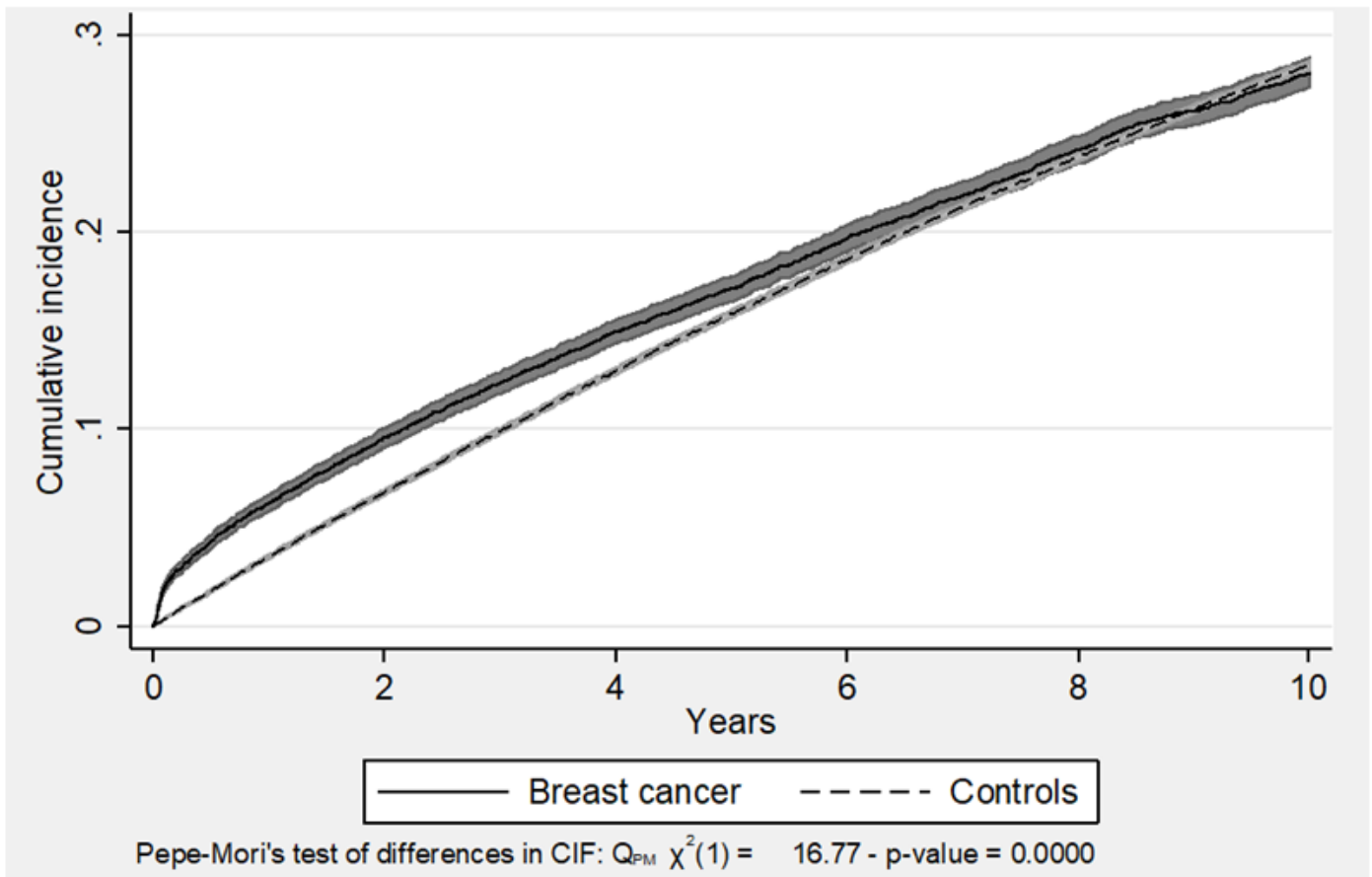

\section{Figure 3}

Cumulative incidence of all CVD diagnoses combined, proportion with at least one CVD diagnosis
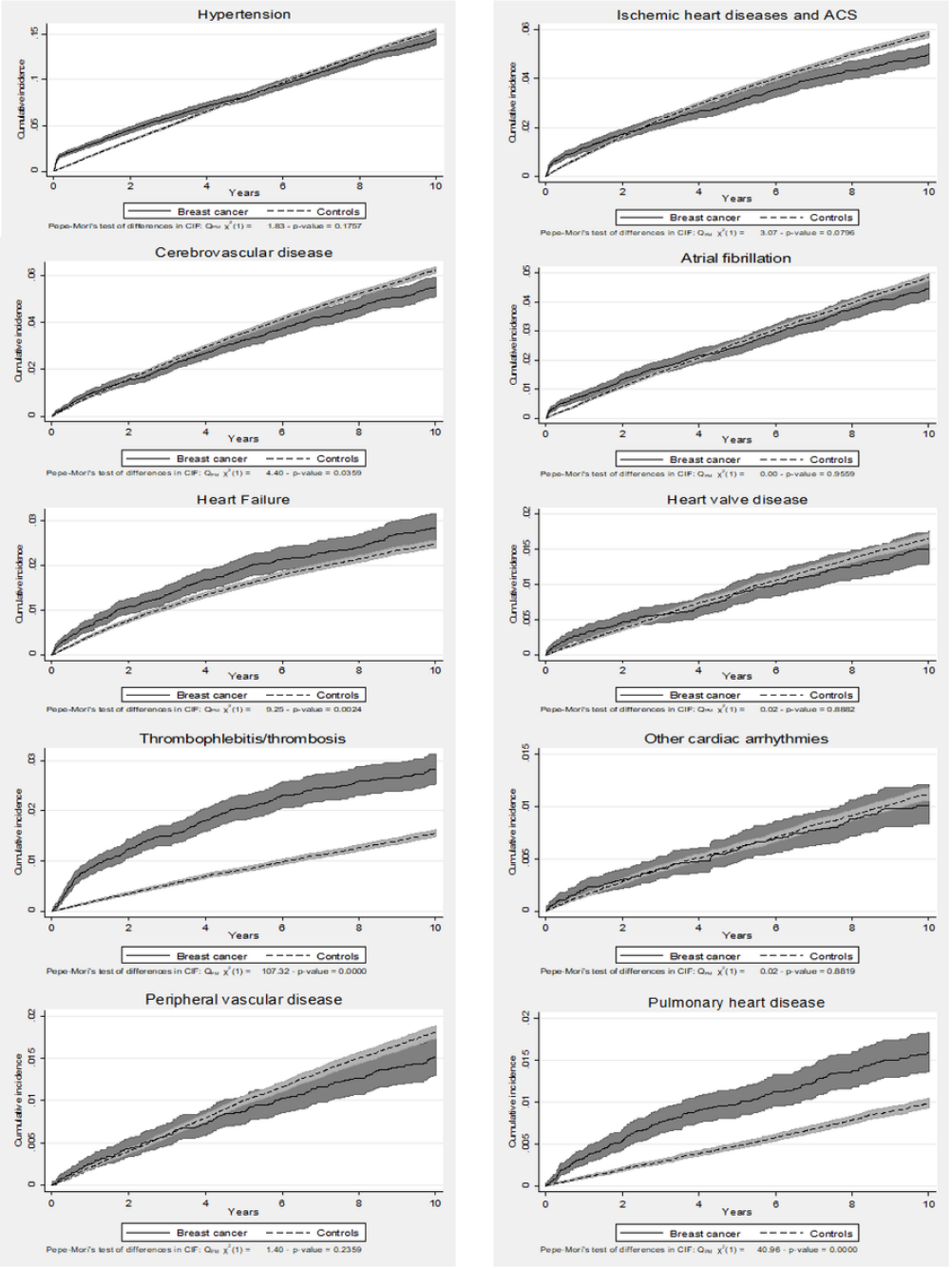
Cumulative incidence of specific types of CVD diagnoses, proportion with CVD diagnosis
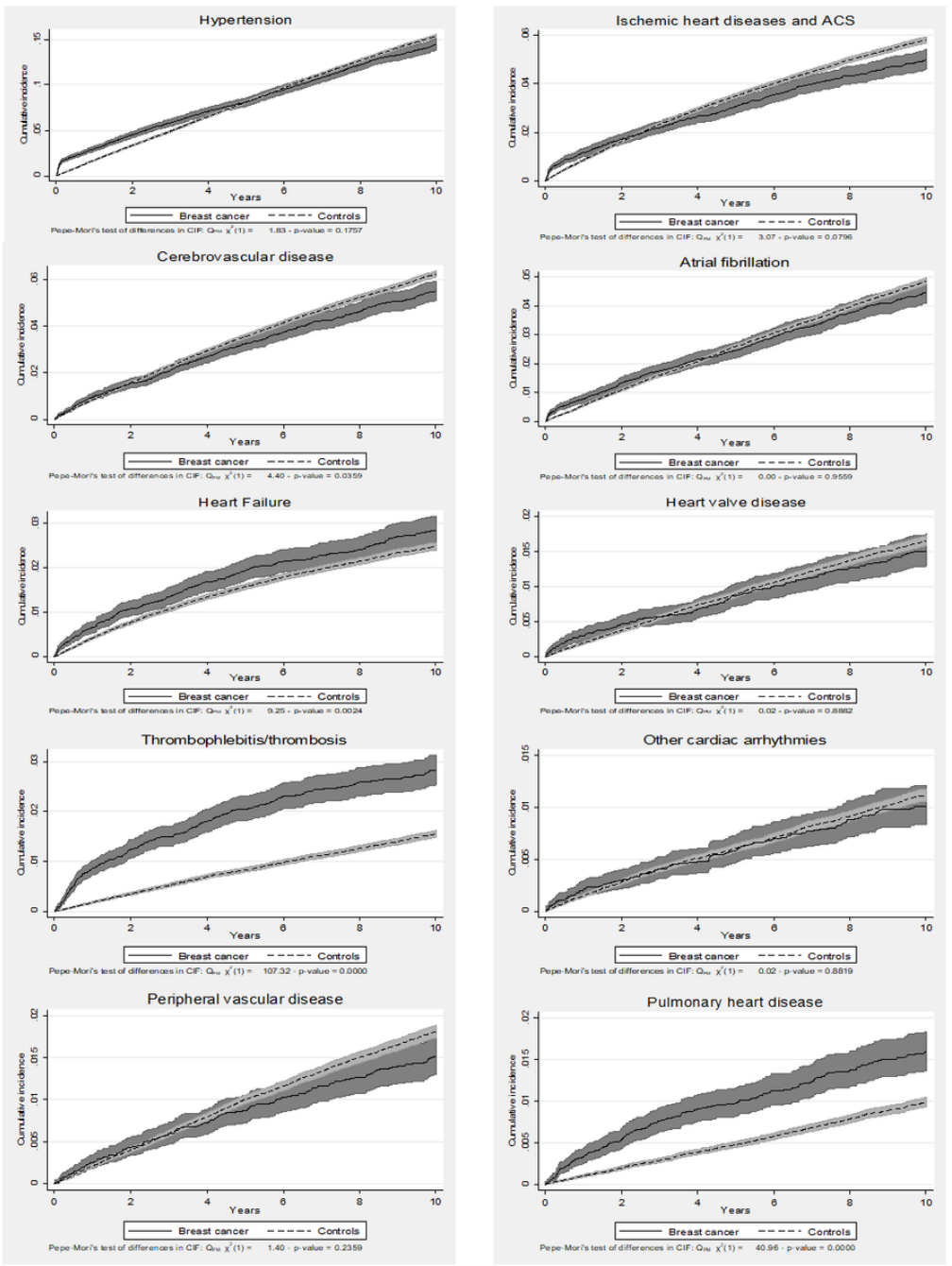

\section{Figure 4}

Cumulative incidence of specific types of CVD diagnoses, proportion with CVD diagnosis
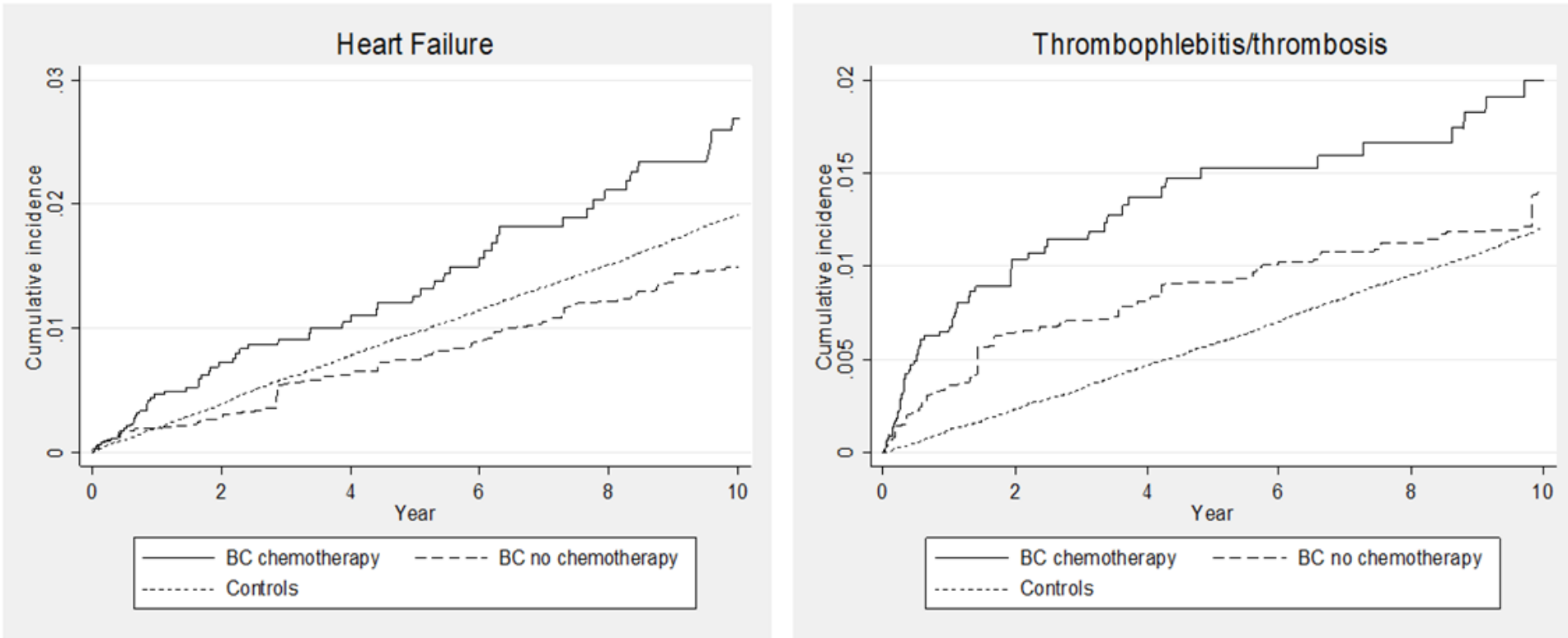

Figure 5 
Cumulative incidence of heart failure and thrombophlebitis/thrombosis after chemotherapy, proportion with diagnosis
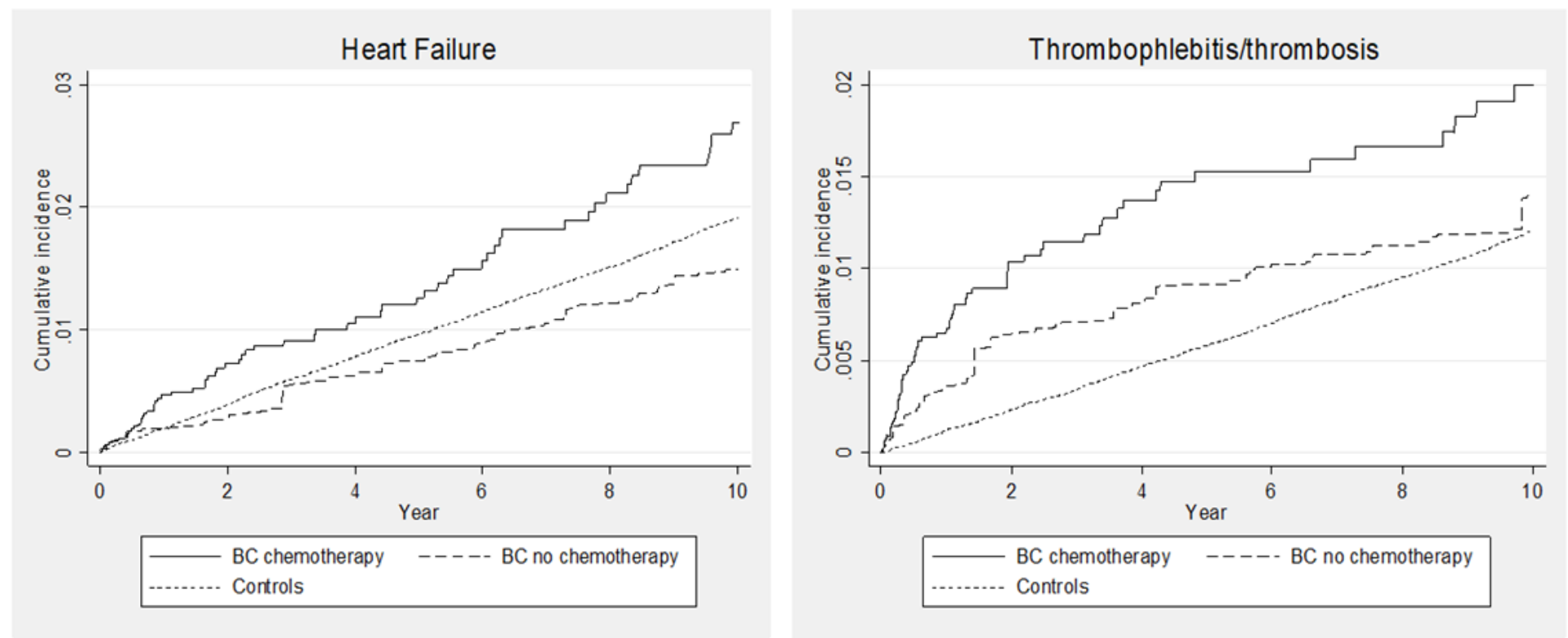

\section{Figure 5}

Cumulative incidence of heart failure and thrombophlebitis/thrombosis after chemotherapy, proportion with diagnosis

\section{Supplementary Files}

This is a list of supplementary files associated with this preprint. Click to download.

- renamedd9177.pdf

- renamedd9177.pdf 